\title{
Commonplace
}

\section{Things with Ashley Farley, Program Officer of Knowledge and Research Services at the Bill and Melinda Gates Foundation}

\section{Ashley Farley}

Published on: Apr 29, 2021

License: Creative Commons Attribution 4.0 International License (CC-BY 4.0). 
I've been thinking about "open" every day for almost 6 years since I first learned about the open access movement as a budding librarian (I use the word "open" as an umbrella term to cover open access, open data, open science, and open research). It is hard not to stay steeped in the nuances of open such as copyright or licensing or various publisher fees or all the colors of the open access rainbow (or minerals?). So, I welcomed this exercise as an opportunity to keep my eye out for interesting, fun or innovative ways to live open practices.

\section{1) The Data Horror Escape Room}

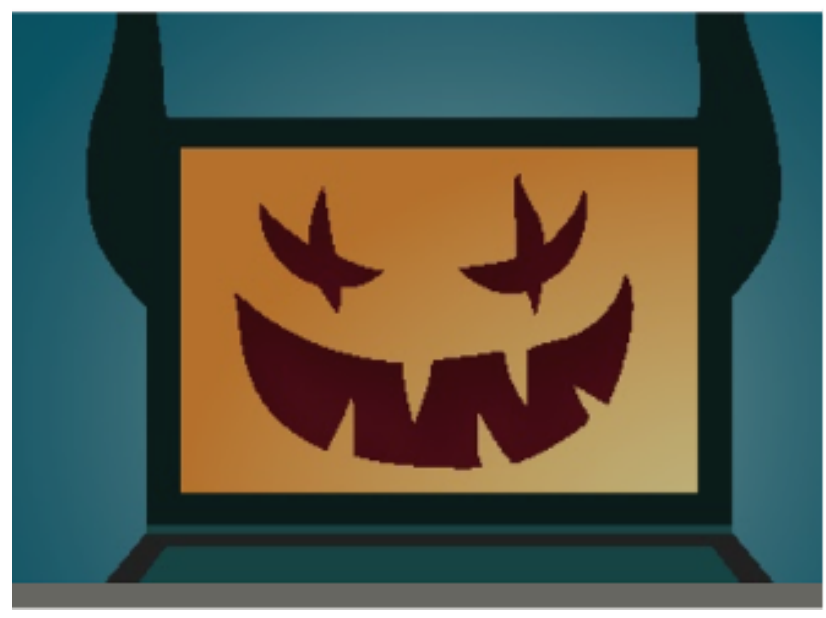

The thought of data management might make one scream or feel trapped in a maze. But what better way to explore the subject of creating data managements plans than through a virtual spooky escape room game? In the Data Horror Escape Room, you will make choices and solve puzzles all while learning a bit more about data sharing. 


\section{2] Which Costs More?}

\section{Which Costs More?}

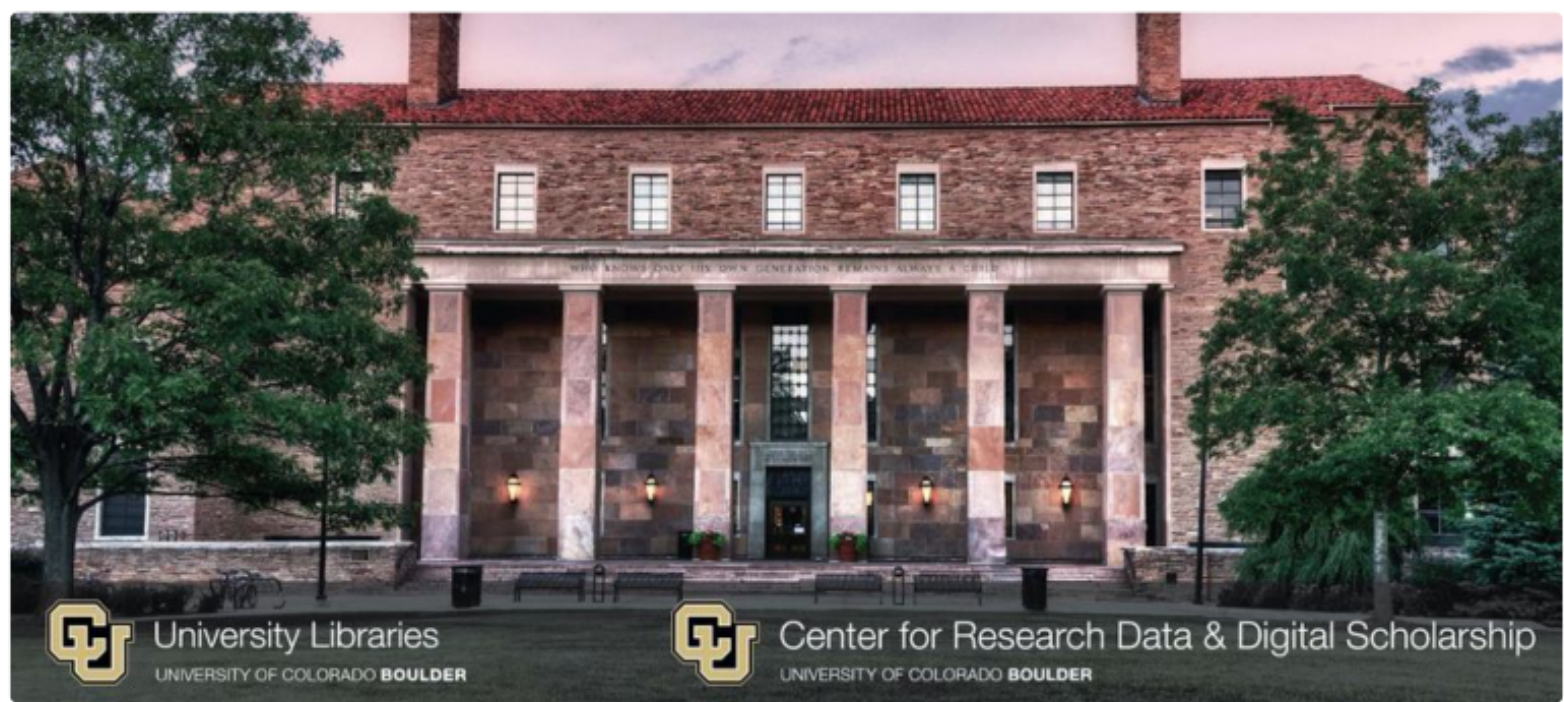

Test your knowledge of the value of CU Boulder Libraries resources

\section{TAKE QUIZ}

Having a gamified way to conceptualize large sums of money can be very helpful. I

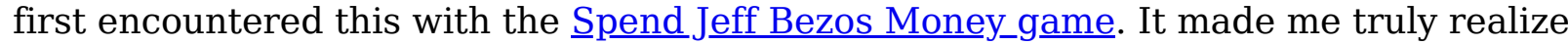
that I cannot comprehend this amount of wealth. It really helps to put things into perspective. When playing "Which Costs More?" keep track of how many times you find yourself surprised by the answer of what may or may not cost more than an institutional subscription. 


\section{3| Open Access Button Levels Up to OA.Works}

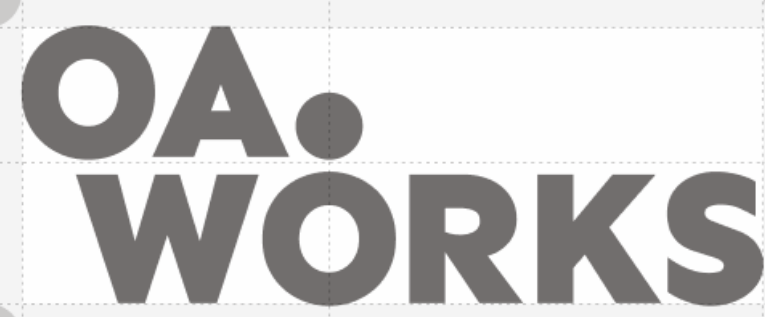

Open Access Button has been a friendly face in the open community for the past 8 years and have been invaluable in connecting libraries, researchers, and the public with access to knowledge. It is a great pleasure to see that the organization has leveled up and rebranded as OA.Works. It's succinct, strong, and salient. I believe the new name will further solidify their important role in the community.

\section{4| Reproducibility Self-Assessment}

I have been a long time fan of the work that Protocols.io and the Public Library of Science $(\underline{P L O S})$ in their efforts to improve access and reliability of research. The Reproducibility Self-Assessment is a really cool tool to help researchers think about the sharing and replicability of their data. It can often feel overwhelming, as we with the Data Horror Escape Room, and this assessment can be a quick way to focus on taking steps in the right direction. 


\section{5| Open Science Stories Podcast}

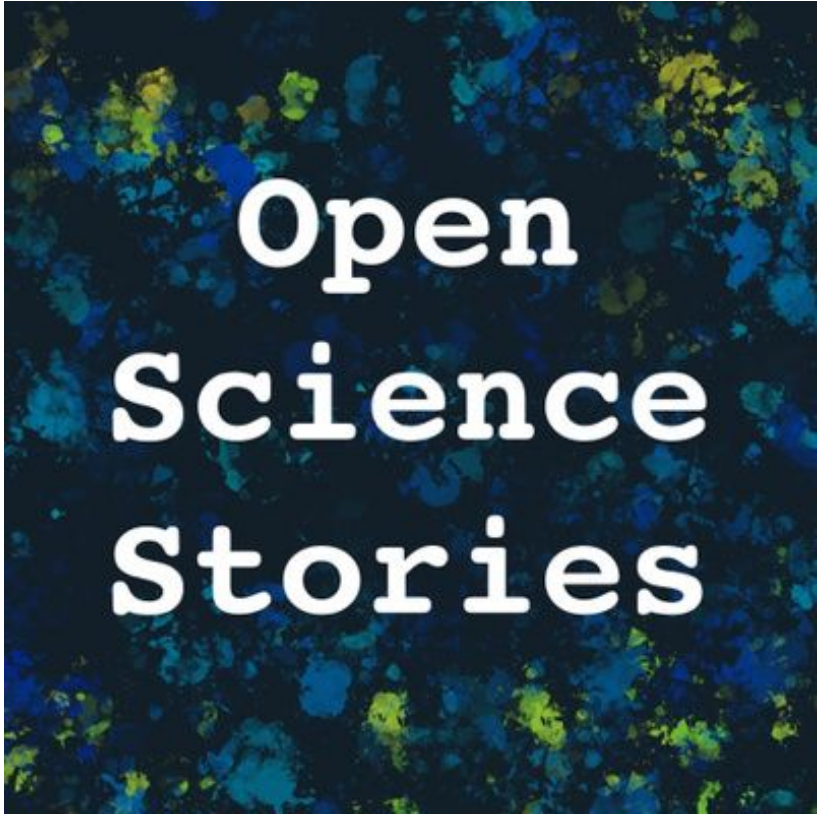

We all know it can be hard to stay updated in our field of interest and open knowledge and research dissemination is no different. One of my favorite fun ways to keep informed is to listen to a podcast. I've recently discovered "Oppen Science Stories" hosted by Heidi Seibold. In just under 10 minutes Open Science Stories covers the latest developments in open in a quick and effective way. I hope that these 5 Things can help provide the energy needed to keep galvanized to continue to practice and learn more about all things open. Feel free to share your favorites in the comment section!

-Ashley Farley 
>>> Subscribe to receive 5 Things to Think About from a different curator in your inbox each $\underline{\text { month }}<<<$ 